\title{
Simulation numérique du phénomène de squat avec un modèle de couplage
}

\author{
Nisrine ALDERF ${ }^{1}$, Emmanuel LEFRANCOIS $^{1}$, Philippe SERGENT ${ }^{2}$, Pierre \\ DEBAILLON $^{2}$ \\ ${ }^{1}$ Université de Technologie de Compiègne, Laboratoire Roberval, UMR 6253 \\ BP 20529, 60205 Compiègne, France \\ nisrine.alderf@utc.fr \\ ${ }^{2}$ CETMEF, 2 Boulevard Gambetta, BP 60039, 60321 Compiègne, France \\ Philippe.Sergent@equipement.gouv.fr
}

\section{Résumé :}

Dans cet article nous nous intéressons à la modélisation numérique par éléments finis du phénomène de surenfoncement dynamique d'un navire (squat). Il est proposé une chaîne de traitement numérique modulaire et donc interchangeable. Celle-ci permet d'étudier l'interaction entre un écoulement bidimensionnel (potentiel) à fonds variables et à surface libre avec prise en compte de la dynamique d'un navire. Le modèle de squat dynamique proposé est utilisé pour valider un modèle analytique basé sur l'extension de la théorie 1D de Schijf (SCHIJF, 1949) aux effets dynamiques en mettant en évidence des positions d'équilibre du navire (stable et instable).

\begin{abstract}
:
In this article we are interested in numerical modelling of "dynamic" phenomenon of squat by finite element analysis. It proposes a modular set of numerical data processing and therefore interchangeable. This model enables the study of the interaction between a two-dimensional flow (potential) at variable depth and free surface with taking into account the dynamics of a ship.

The proposed model has been used to validate a stability model based on the extension of the 1D theory of Schijf (SCHIJF, 1949) to the dynamic effects by pointing out positions of stability for ship.
\end{abstract}

\section{Mots-clés :}

Squat - Déformation de maillage - Dynamique de navire - Eléments finis Ecoulement potentiel - Couplage fluide-structure - Système masse-ressort. 


\section{Introduction}

Au cours de la décade qui suivit les années 60, un certain nombre d'échouage des navires se produisaient par petit fond, à ceci, s'ajoutent immanquablement les risques d'impact écologique (liés essentiellement aux navires pétroliers), un bilan économique lourd (montant de la restauration du navire, dédommagement...) et parfois humain.

Considérons un navire à l'équilibre statique, avec un tirant d'eau T. Lorsque ce navire est mis en mouvement la vitesse du navire donne naissance à un surenfoncement supplémentaire par rapport à $\mathrm{T}$. Il s'en suit, D'après le théorème de Bernoulli, une diminution de la pression globale de l'eau sur la carène qui, étant moins portée, s'enfonce. En enfonçant, l'assiette du navire peut être modifiée. Le surenfoncement du navire avec ou sans variations d'assiette est dénommé squat (BARRASS, 2006; DEBAILLON, 2005 ; HERVIEU, 1993).

Afin de simuler le phénomène du squat, nous proposons une chaîne de traitement numérique modulaire et interchangeable, articulée autour de quatre modèles principaux: modèle fluide, modèle structure, modèle surface libre et modèle déformation de maillage. Un modèle analytique intermédiaire est présenté pour compléter le modèle de SCHIJF (SCHIJF, 1949) de l'aspect dynamique et permettre une étude comparative avec la chaîne de traitement numérique.

L'article est composé de trois parties, dans la deuxième partie nous présentons le modèle analytique d'équilibre dynamique. La chaîne de traitement numérique est présentée dans la troisième partie. La quatrième partie montre les résultats numériques obtenus. Une conclusion avec perspectives conclue ce papier.

\section{Modèle analytique d'équilibre dynamique}

L'équilibre dynamique du navire est régi par le principe fondamental de la dynamique (PFD) écrit sous la forme suivante (voir figure 1) :

$m \frac{d^{2} \omega}{d t^{2}}=-m g+\int P \vec{n} \cdot \overrightarrow{\mathrm{e}}_{\mathrm{z}} d s \quad$ avec $\quad m=\rho . L p p . T$

où :

$\omega=$ déplacement du navire suivant l'axe $\mathrm{z}$.

$\mathrm{m}=$ la masse du navire par unité de largeur.

$T=$ tirant d'eau.

$\mathrm{P}=$ pression.

$\rho=$ masse volumique d'eau

$\mathrm{g}=$ accélération de gravité.

Lpp $=$ longueur entre perpendiculaire. 
La pression sous la carène est supposée homogène.

La conservation des débits permet de calculer la vitesse sous la carène :

$V(\omega)=V_{\infty} \frac{H}{H-T+\omega}$

où :

$V_{\infty}=$ vitesse d'avancement du navire (vitesse de l'écoulement (principe d'inversion (HERVIEU, 1993)).

La pression sous la carène s'écrit d'après Bernoulli par :

$P_{2}=P_{a t m}+\rho \cdot g \cdot(T-\omega)+0.5 \cdot \rho \cdot V_{\infty}^{2}\left(1-\left(\frac{H}{H-T+\omega}\right)^{2}\right)$

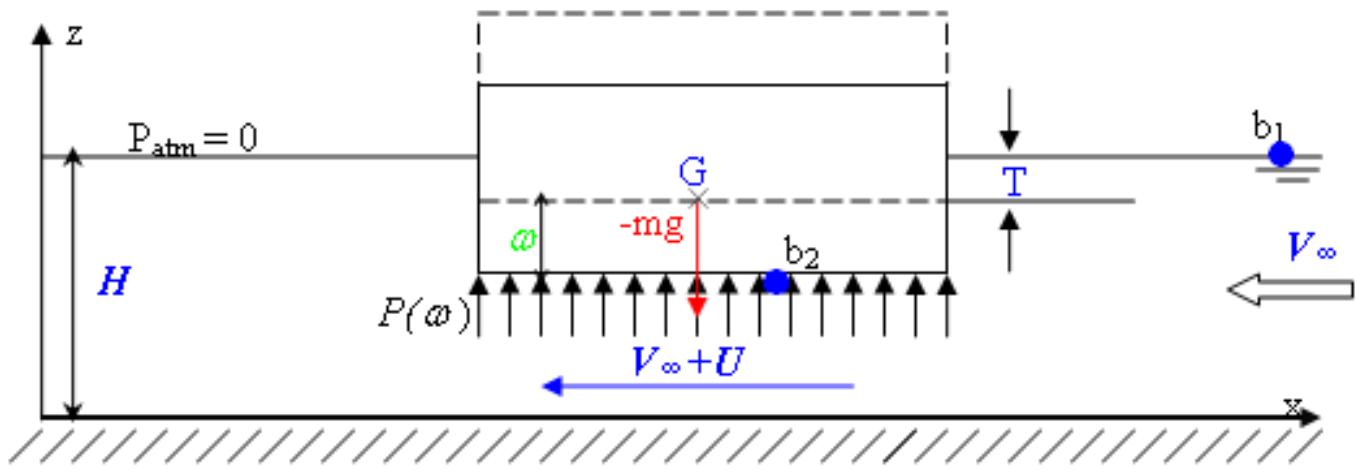

Figure 1. Modèle d'équilibre dynamique et nomenclature

La relation (1) s'écrit finalement :

$m \frac{d^{2} \omega}{d t^{2}}=\frac{\rho \cdot L p p}{(H-T+\omega)^{2}} \times$

$\left(-g \omega^{3}+\left(-2 g(H-T)+\frac{1}{2} V_{\infty}^{2}\right) \omega^{2}+\left(-g(H-T)^{2}+V_{\infty}^{2}(H-T)\right) \omega+\frac{1}{2} V_{\infty}^{2}\left((H-T)^{2}-H^{2}\right)\right)$

\subsection{Analyse de stabilité du navire}

Le second membre peut être réécrit sous une forme polynomiale admettant trois racines $\omega_{1}, \omega_{2}$ et $\omega_{3}$ (correspondant chacune à une position d'équilibre du navire) telle que :

$m \frac{d^{2} \omega}{d t^{2}}=\frac{-g \cdot \rho \cdot L p p}{(H-T+\omega)^{2}} \times\left(\left(\omega-\omega_{1}\right)\left(\omega-\omega_{2}\right)\left(\omega-\omega_{3}\right)\right)$ 
Multiplier l'équation (4) par la vitesse verticale du navire $\frac{d \omega}{d t}$ permet après intégration, de définir les énergies cinétique et potentielle du navire :

$E_{c}=\frac{1}{2} m\left(\frac{d \omega}{d t}\right)^{2} \quad$ et $\quad E_{p}=-\int\left(\frac{\rho \cdot L p p}{(H-T+\omega)^{2}}\right) \times f(\omega) d \omega$

où :

$f(\omega)=-g \omega^{3}+\left(-2 g(H-T)+\frac{1}{2} V_{\infty}^{2}\right) \omega^{2}+\left(-g(H-T)^{2}+V_{\infty}^{2}(H-T)\right) \omega+\frac{1}{2} V_{\infty}^{2}\left((H-T)^{2}-H^{2}\right)$

La stabilité des positions d'équilibre est contrôlée par le signe de la dérivée seconde de l'énergie potentielle :

- une valeur positive traduit une position stable (cas de $\omega_{1}$ ).

- une valeur négative traduit une position instable (cas de $\omega_{2}$ ).

Il est possible de montrer que la racine stable $\omega_{3}$ n'a pas de sens physique.

\subsection{Vitesse critique au surenfoncement}

Il existe une vitesse critique à partir de laquelle on passe d'un régime de navigation stable à un régime critique (instable) où $\omega_{1}$ et $\omega_{2}$ deviennent égales complexes et conjuguées (voir paragraphe 4.4).

\section{Modèle éléments finis 2D de couplage carène-écoulement}

Ce modèle est architecturé autour d'une chaîne de traitement numérique modulaire et donc interchangeable. Ce modèle est articulé autour de cinq modèles principaux :

- un modèle fluide: basé sur une approche d'écoulement potentiel par éléments finis 2D linéaire (DHATT et al, 2005).

- un modèle structure: basé sur la résolution de la dynamique d'une structure avec un schéma de résolution explicite en temps.

- un modèle de surface libre: basé sur l'approximation de Bernoulli (DEBAILLON, 2005).

- un modèle de déformation du maillage fluide (basé sur une approche de type pseudo-matériaux avec technique de sous mailles) pour permettre d'assurer une adéquation entre la position du navire et les frontières du maillage fluide) (JOHNSON \& TEZDUYAR, 1994; LEFRANCOIS, 2007). 
- un modèle de variation du fond dû à l'écoulement relatif de la carène.

\subsection{Domaine d'étude}

Nous nous plaçons dans le cas d'un écoulement relatif par rapport au navire qui est donc supposé fixe. Nous considérons le cas général d'un canal à fond mobile, avec un flux constant de droite vers la gauche de vitesse $V_{\infty}$ (voir figure 2).

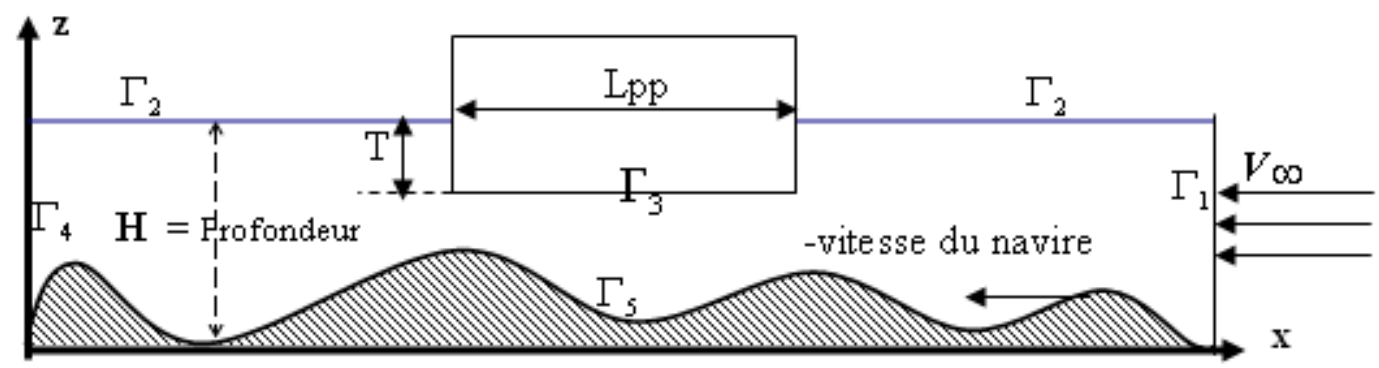

Figure 2. Définition du domaine

Les conditions aux limites sont de type vitesse imposée en entrée $\Gamma_{1}$, glissement sur le navire $\Gamma_{3}$ et le fond $\Gamma_{5}$ et potentiel imposé sur $\Gamma_{4}$ pour assurer l'unicité de la solution.

\subsection{Schéma de couplage}

Le schéma de couplage retenu est illustré figure 3.

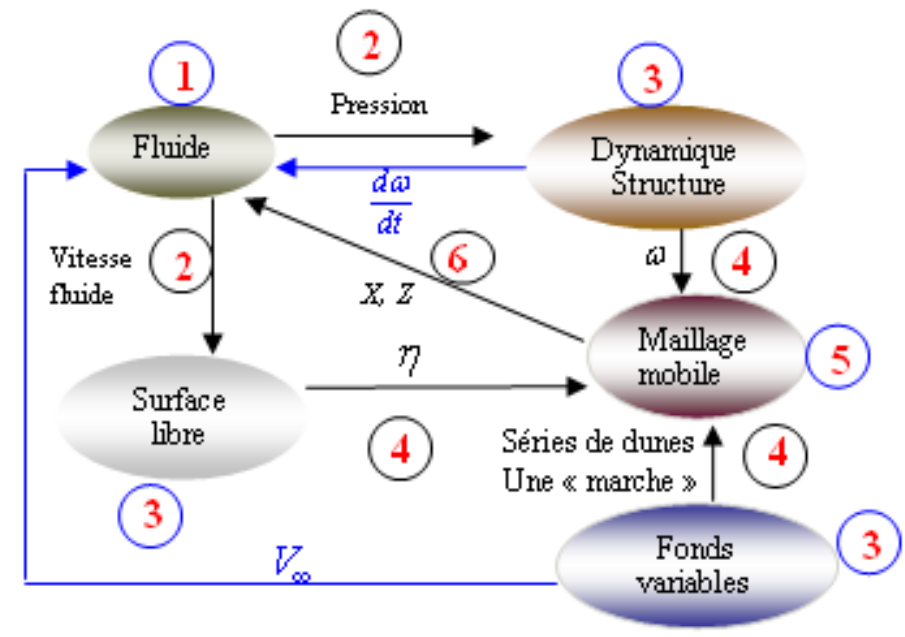

Figure 3. Schéma du couplage fluide-structure

Le code fluide commence par résoudre l'écoulement potentiel et fournit le champ de pression au code de structure. Ce dernier déduit du PFD le champ de déplacement et les vitesses de la carène et les transmet au code de déformation du 
maillage qui transmet en retour, les coordonnées du maillage déformé au code fluide. Il y a un retour d'informations en termes de vitesses, puisque la vitesse de déplacement du navire est intégrée dans le calcul de l'écoulement via l'usage d'éléments de contour sur la carène et le fond qui "poussent" le fluide pour assurer la conservation de débit.

\section{$4 \quad$ Résultats}

\subsection{Squat statique et positions d'équilibre}

Afin de retrouver les résultats de squat statique, à savoir la position d'équilibre stable $\omega_{1}$, le code de couplage est volontairement amorti pour le calcul de la dynamique du navire afin d'annuler la réponse dynamique de la structure et obtenir ainsi son enfoncement statique.

Pour les paramètres donnés dans le tableau (1) avec la longueur du canal L, nous avons comparé les valeurs du squat «statique » calculées par le code de couplage avec celles prédites par le modèle d'équilibre (équation (5)). Les résultats (voir figure 4) sont exprimés en fonction du coefficient de blocage $C_{b}$ défini par le rapport $\mathrm{T}$ sur $\mathrm{H}$. La figure 4 montre la bonne adéquation entre les deux modèles.

Tableau 1 : Paramètres

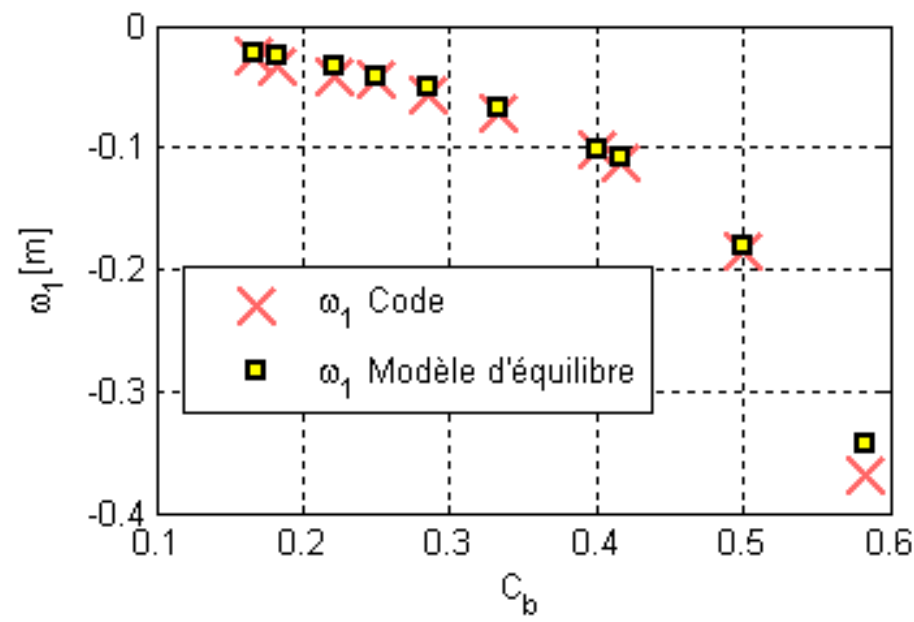

\begin{tabular}{l}
\hline Paramètres du navire \\
\hline$L p p=20[\mathrm{~m}]$ \\
\hline Paramètres du canal \\
\hline$V_{\infty}=1[\mathrm{~m} / \mathrm{sec}]$ \\
\hline$L=100[\mathrm{~m}]$ \\
\hline Paramètres du schéma \\
\hline$\Delta t=0.1[\mathrm{sec}]$ \\
\hline$\omega_{0}=0.005[\mathrm{~m}]$ \\
(enfoncement initial)
\end{tabular}

Figure 4. Confrontation modèle couplé et

d'équilibre : position de squat

\subsection{Fréquence de réponse et analogie masse-ressort}

Un test de validation a été mené pour comparer la période de réponse naturelle du navire avec celle du modèle d'équilibre (voir figure 5) pour les paramètres donnés dans le tableau (2). 
Tableau 2 : Paramètres

\begin{tabular}{|l|l|l|}
\hline Paramètres du navire & Paramètres du canal & Paramètres du schéma \\
\hline$T=2[\mathrm{~m}]$ & $V_{\infty}=1[\mathrm{~m} / \mathrm{s}]$ & $\Delta t=0.1[\mathrm{sec}]$ \\
$H=6[\mathrm{~m}]$ & $L=100[\mathrm{~m}]$ & $\omega_{0}=0.005[\mathrm{~m}]$ \\
$L p p=20[\mathrm{~m}]$ & & \\
\hline
\end{tabular}
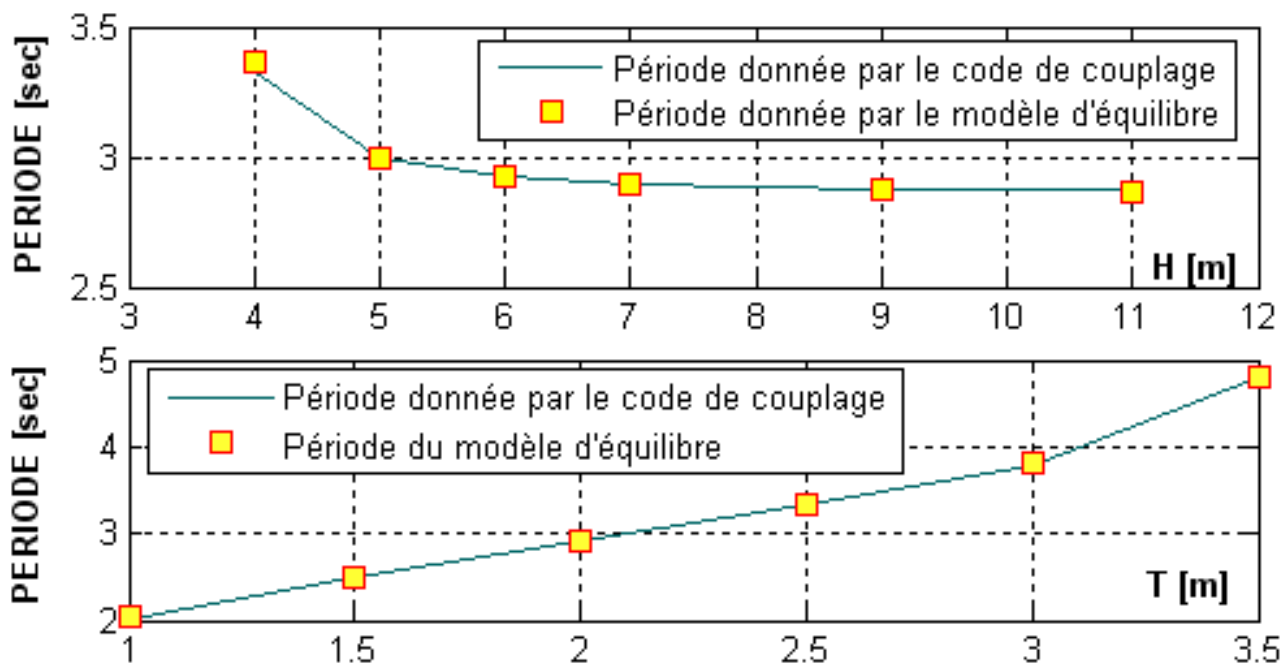

Figure 5. Confrontation modèle couplé et d'équilibre : période du navire

La période tracée dans la figure (5) est obtenue en faisant une moyenne arithmétique sur les cinq premières périodes mesurées dans le signal de $\omega$ enregistré au cours du temps.

En augmentant T, la période augmente en raison de l'augmentation de la vitesse au dessous de la carène, dans ce cas la partie hydrodynamique de l'équation de Bernoulli commence à jouer un rôle important par rapport à celui de la partie hydrostatique. La même explication peut être apportée dans le cas où $\mathrm{H}$ diminue. On remarque que pour les petites vitesses de déplacement du navire, la période de réponse du navire obtenue par le modèle couplé et le modèle d'équilibre est analogue à celle d'un système masse ressort donnée par :

PERIODE $=2 \pi \sqrt{\frac{T}{g}}$

\subsection{Réponse dynamique au passage d'une série de dunes}

Dans cet exemple nous montrons que la dynamique du navire peut être générée Dans cet exemple nous montrons que la dynamique du navire peut être générée par le passage d'une série de dunes suivant les paramètres du navire et des dunes. Selon les valeurs de la longueur de dune, de celle du navire (Lpp) et de la vitesse d'écoulement relatif, on peut observer soit une réponse quasi- statique du navire 
(voir figure 6), soit une réponse dynamique (voir figure 7) pour les paramètres donnés dans le tableau (3).

Tableau 3 : Paramètres
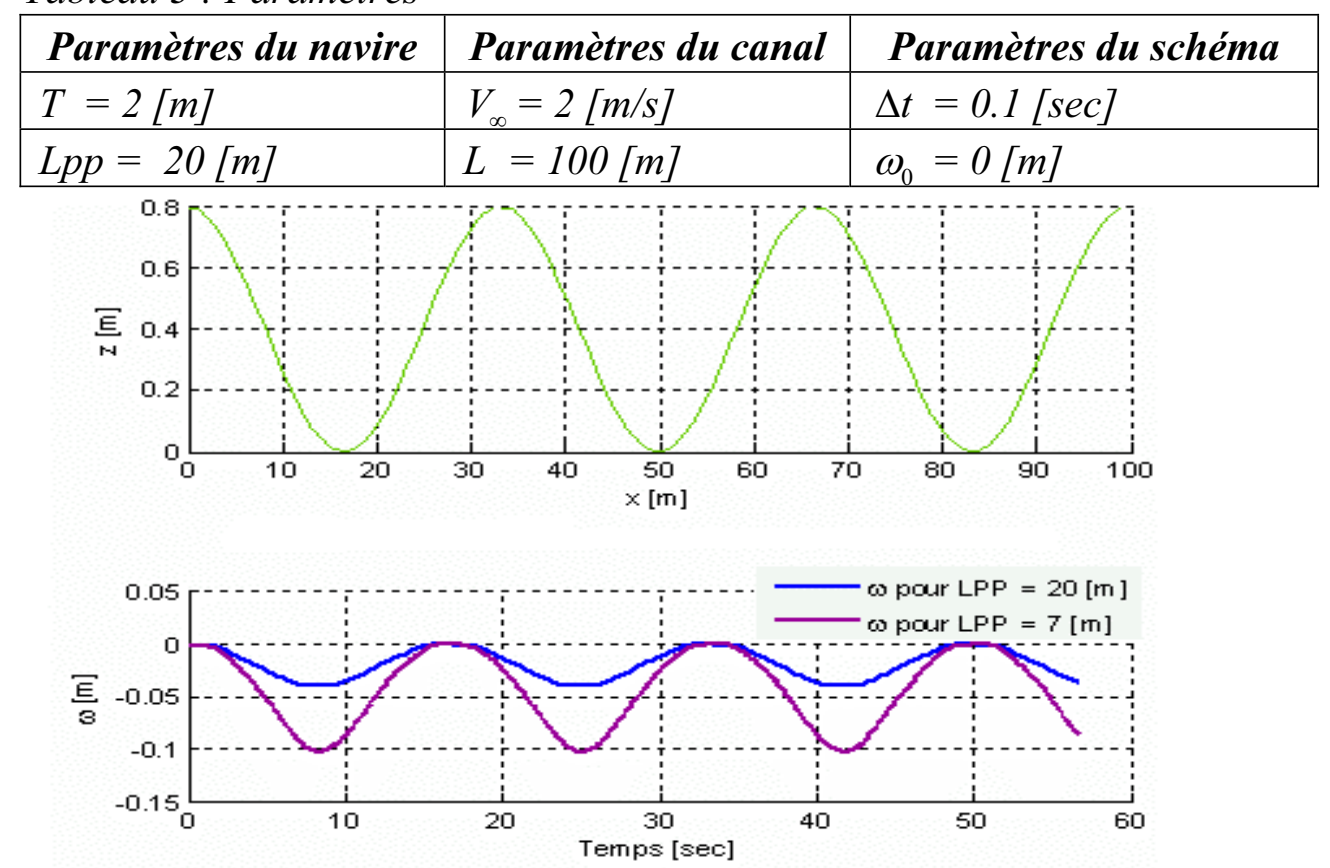

Figure 6. Réponse statique d'un navire au passage d'une série de dune

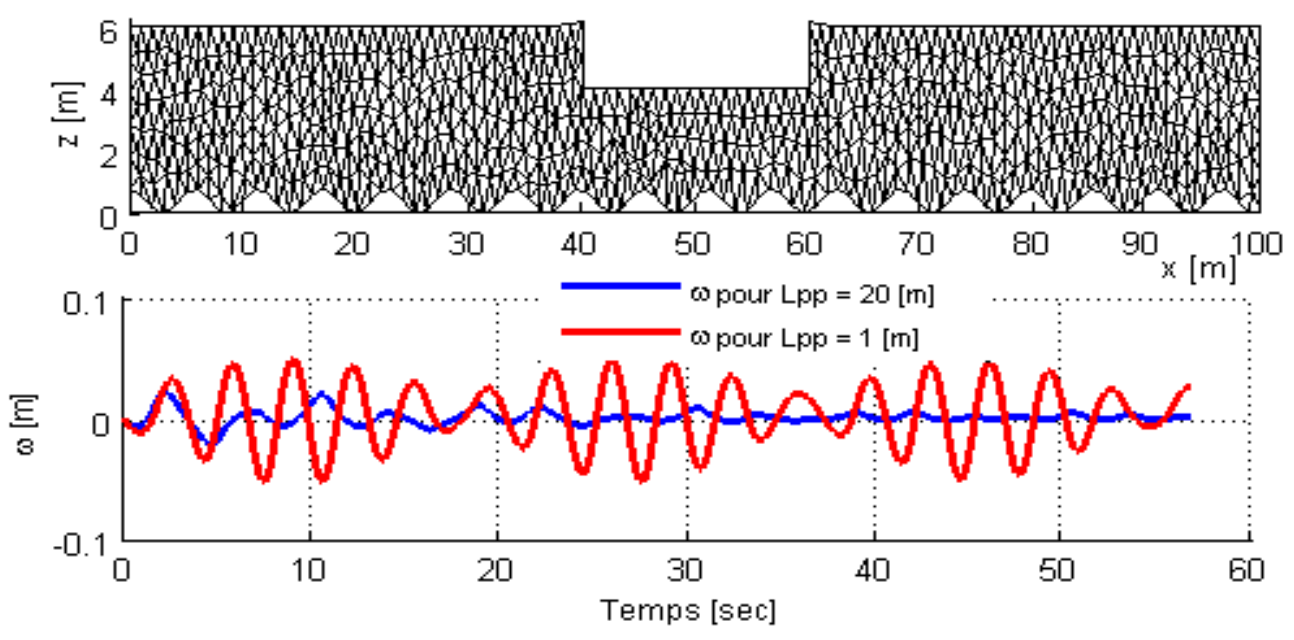

Figure 7. Réponse dynamique d'un navire au passage d'une série de dune

\subsection{Influence de variation de fond sur la stabilité de navire}

Nous avons mentionné dans le paragraphe (2 .2) l'existence d'une vitesse critique fonction de la profondeur d'eau. Une variation brutale de cette profondeur, (exemple du passage d'une «marche » de hauteur $\mathrm{A}_{\text {marche }}$ ) peut donc 
entraîner un changement de la valeur de vitesse critique et rendre instable l'équilibre initialement stable du navire. Nous avons donc simulé la variation d'un fond par le passage d'une marche pour les paramètres donnés dans le tableau (4).

Tableau 4 : Paramètres

\begin{tabular}{|l|l|l|}
\hline Paramètres du navire & \multicolumn{1}{|c|}{ Paramètres du canal } & Paramètres du schéma \\
\hline$T=2[\mathrm{~m}]$ & $H=6[\mathrm{~m}]$ & $\Delta t=0.1[\mathrm{sec}]$ \\
$\mathrm{Lpp}=20[\mathrm{~m}]$ & $L=100[\mathrm{~m}]$ & $\omega_{0}=0[\mathrm{~m}]$ \\
$V_{\infty}=1[\mathrm{~m} / \mathrm{s}]$ & $A_{\text {marche }}=2.8[\mathrm{~m}]$ & \\
\hline
\end{tabular}

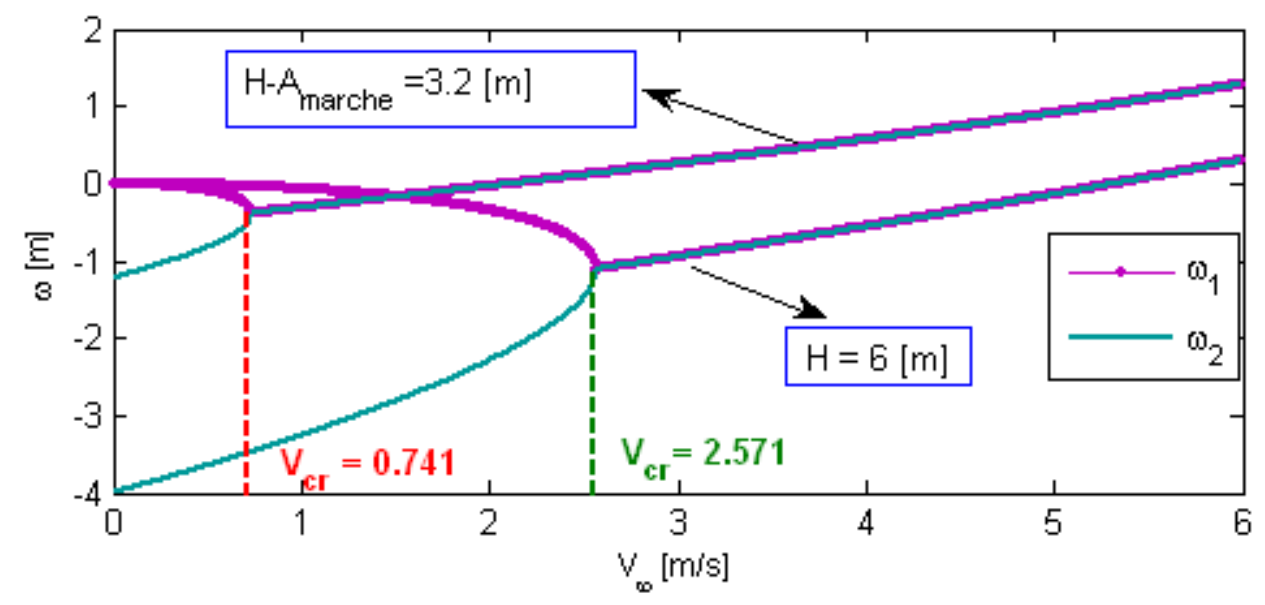

Figure 8. Evolution de positions d'équilibre avec et sans marche
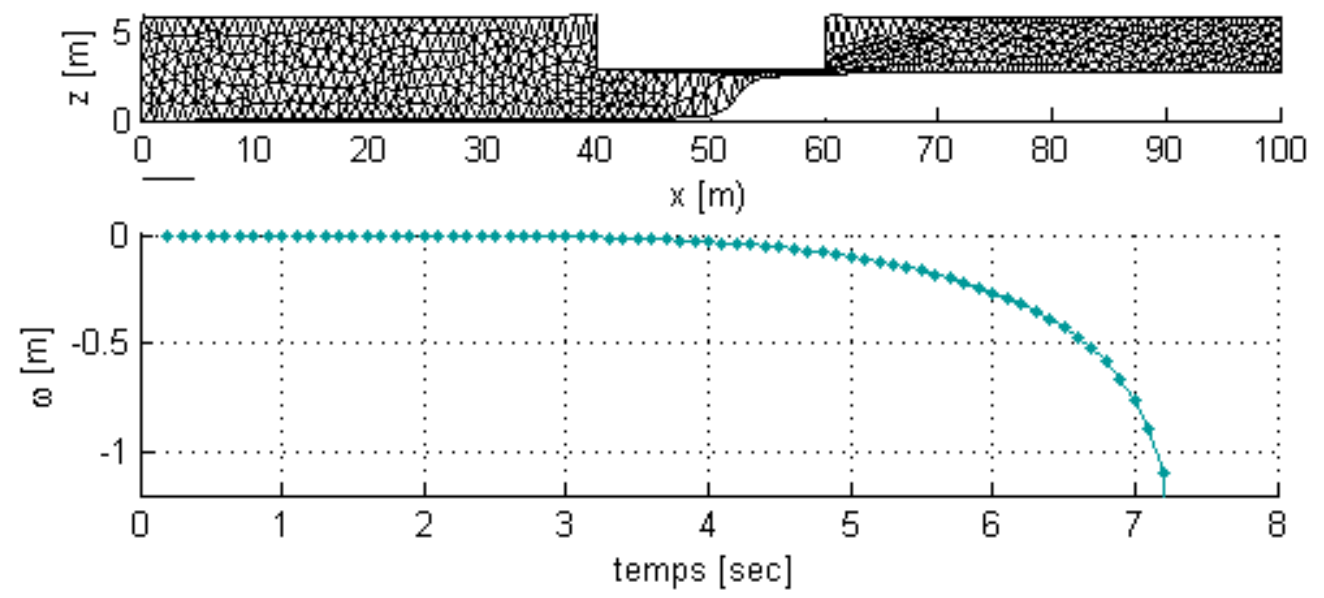

Figure 9. Instabilité du navire au passage d'une marche.

Nous illustrons figure (8) les prédictions des positions d'équilibre $\omega_{1}$ et $\omega_{2}$ données par le modèle de stabilité avec et sans marche. On observe qu'après le passage de la marche, la vitesse critique de surenfoncement passe de $2.571 \mathrm{~m} / \mathrm{s}$ à $0.741 \mathrm{~m} / \mathrm{s}$. La figure (9) illustre les résultats du modèle de couplage en 
confirmant les prédictions d'instabilité au passage de la marche : la figure de haut représente le navire au moment de l'impact avec le fond, la figure du bas illustre la réponse en enfoncement.

\section{Conclusion}

Dans cet article le phénomène de squat a été simulé par un modèle d'équilibre basé sur le PFD et un modèle numérique de couplage fluide-structure, les deux modèles sont confrontés en mettant en évidence plusieurs positions d'équilibre du navire (stable et instable). Il a été montré que, pour les petites vitesses, la période de réponse naturelle du navire est analogue à celle d'un système masse-ressort. L'influence de variation de fond sur le régime de navigation du navire a été analysée en montrant que la dynamique du navire peut être générée et entretenue par le passage sur une série de dunes et en confirmant les prédictions d'instabilité due au passage d'une «marche ».

Comme perspectives, nous proposons la simulation des effets visqueux en déterminant un coefficient d'amortissement permettant l'extension du modèle fluide à un modèle de type Navier Stokes et en 3D.

\section{Références bibliographiques}

1 BARRASS C.B., DERRETT D.R. (2006). Ship stability for master and mates. pp 324-336.

2 DEBAILLON P. (2005). Système de modélisation de l'enfoncement dynamique des bateaux. Thèse, UTC.

3 JOHNSON A.A., TEZDUYAR T.E. (1994). Mesh update strategies in parallel element computations of flow problems with moving boundaries and interfaces, Comput. Methods APPL. Mech. Engrg. 119, pp 73-94

4 DHATT. G., TOUZOT. G., LEFRANCOIS E. (2005). Méthode des éléments finis, Lavoisier, pp 21-73

5 HERVIEU R. (1993). Le surenfoncement des navires : introduction à l'hydrodynamique navale, Masson, pp 5-12

6 LEFRANCOIS E. (2007). A simple mesh deformation technique for fluidstructure interaction based on a submesh approach. Int. J. Numer. Meth. Engng.

7 SCHIJF J. B. (1949). 17eme congrès de la navigation internationale. S.I. C.2. 